\title{
Training in echocardiography
}

\author{
Authorship and \\ consultation \\ These guidelines were \\ developed by the \\ Education and Training \\ Subcommittee of the \\ British Society of \\ Echocardiography \\ (BSE): Chairman, Mark \\ Monaghan (King's \\ College Hospital); \\ Valerie Anderson (Royal \\ Lancaster Infirmary); \\ John Chambers (Guy's \\ Hospital); Graham \\ Leech (St George's \\ Hospital); Michael \\ Martin (Stepping Hill \\ Hospital); Petros \\ Nihoyannopoulos \\ (Hammersmith \\ Hospital); Caroline \\ Westgate (Royal \\ Brompton National \\ Heart and Lung \\ Hospital); and Peter \\ Wilde (Bristol Royal \\ Infirmary). Its contents \\ and proposals have been \\ endorsed by the BSE \\ Council. \\ Advice was sought from \\ the British Cardiac \\ Society, American \\ Society of \\ Echocardiography, \\ United Kingdom \\ Association of \\ Sonographers, Society of \\ Cardiac Technicians, \\ British Medical \\ Ultrasound Society, City \\ of Westminister College, \\ and Salford College of \\ Technology.
}

There are no formal training guidelines for doctors, technicians, and others performing echocardiographic studies in the United Kingdom and the Republic of Ireland. As a result the skill and experience of operators performing and reporting echocardiograms to the same level of responsibility vary widely from centre to centre and within individual departments. Recognition of competence at echocardiography is arbitrary both for doctors and technicians. This can place an unfair burden on an individual who may be required to perform an echocardiogram and interpret it without appropriate training, guidance, or experience. Though the day-to-day responsibility of running the echocardiography service may fall upon an appropriately experienced (and in the future possibly accredited) person the BSE believes that overall legal responsibility lies with the consultants in charge of the departments and that the onus is upon them to ensure appropriate training of staff.

The problem of training in echocardiography is compounded by the diversity of courses and institutions available for training. There is wide variation in the topics covered, the balance between theoretical and practical aspects, the quality of teaching delivered, and the standards expected of course attendees. Most courses try to be all-embracing and it is difficult for a student to decide which course is likely to be the most appropriate to his or her needs.

This document proposes a list of topics to be covered in an echocardiographic training schedule and it defines the level of basic knowledge that should be attained before more specific training is undertaken. Currently available courses cover most of the basic topics and it would be desirable to encourage course organisers to relate their training schedules to the BSE syllabus. Echocardiography training courses that cover the syllabus recommendations of the BSE may achieve some form of recognition or accreditation.

For those wishing to have objective proof of their competence, formal assessment of echocardiographic expertise should be available. The assessment would be based upon the BSE syllabus and would help to improve standards. Such provision is in line with recommendations being developed for postgraduate medical and paramedical training and also with accreditation proposals currently being formulated by the Consortium for the Accreditation of Sonographic Education (CASE). This document includes proposals for assessment of both echocardiographers and training courses.

\section{General principles}

The skill and knowledge required to perform and to interpret an echocardiogram are closely interlinked. Those whose primary task is to supervise echocardiographic studies and interpret the significance of the findings must also be trained and adequately skilled in performing the examinations so that they can judge the technical quality and reliability of the data. Conversely, the person performing echocardiographic studies must have not only technical and scanning ability but also must understand the clinical context of the examination. The nature of the disease or lesion dictates which specific views and examination techniques are needed. Unexpected lesions are frequently found in the course of an examination and, unless the echocardiographer has sufficient experience and judgment to be able to evaluate the findings accurately and immediately, adequate data may not be recorded and the results of the study may be inconclusive or misleading.

Everyone involved in the delivery of an echocardiographic service must be skilled both in performing and in reporting clinical studies. Therefore, the training requirements of both medical and paramedical staff are essentially the same though their medico-legal responsibilities may differ.

Before trainees learn the specifics of adult or paediatric echocardiography they require a basic level of background knowledge. This includes an understanding of the physical principles of image formation and Doppler ultrasound; familiarity with the proper and safe use of echocardiographic instrumentation; knowledge of cardiac anatomy, physiology, and haemodynamics, and how these are affected by pathological conditions; and an understanding of the clinical setting of cardiac problems and the likely differential diagnoses. Doctors should already have adequate knowledge of cardiovascular anatomy and abnormalities but they may need training in physics and instrumentation; technical staff, radiographers, and clinical scientists will probably need instruction in cardiac anatomy and pathophysiology.

It is easier to teach and examine the theory of echocardiography than to devise schemes to teach and assess practical skills. The rate at which a trainee becomes skilled in performing and interpreting echocardiographic studies 
Summary of proposed echocardiographic training schedule

Basic level

Basic cardiovascular

anatomy and physiology

Physical principles of ultrasound imaging and Doppler studies

Ultrasound instrumentation

Normal views and scanning

Common cardiac

abnormalities

Introduction to clinical

Doppler studies

Proficiency level

Core

Advanced cardiac anatomy and pathophysiology

Principles of normal flow

patterns and Doppler

patterns and Dopp

Introduction to

transoesophageal

transoesophageal

Plus either

Adult echocardiography and Doppler option

or

Paediatric echocardiography and Doppler option

Specialised level ${ }^{\star}$

Choice from:

Choice from: techniques, views and abnormalities

Intravascular imaging and Doppler studies

Advanced imaging and Doppler topics

Advanced instrumentation (quality assurance, calibration, specifying equipment, artefacts etc.)

Advanced cogenital heart disease

Fetal cardiac studies

$\star$ Subject to continued review will depend upon previous training, knowledge and technical abilities, the number and type of patients examined, and the teaching skills of those in the department. It is highly desirable for a trainee to work under the direction of experienced echocardiographers in a department where many studies are being performed. This may take a considerable time. A useful way of ensuring that training and experience are adequate is for trainees to record in a log-book all the studies performed and/or interpreted. This procedure was adopted by the Royal College of Radiographers for their Diploma in Medical Ultrasound and by the British Cardiovascular Intervention Society, which is affiliated to the British Cardiac Society. It ensures that an individual receives the required amount of practical experience and provides proof of this. The training record should include a videotape of cases performed by the echocardiographer.

When it is difficult to obtain suitable experience, guidance, and training, facilities should be provided at reference sites. The BSE would like to accredit as many existing echocardiography departments as possible as reference sites so that access to high quality training is made widely available. The BSE expects that reference sites will organise assessment exercises and provide mentors for trainees. Accreditation of reference sites has yet to be fully defined and under the NHS internal market, reference sites may have to charge for their services.

It is unrealistic to expect that all trainees in echocardiography should achieve an identical level of competence and this is neither appropriate nor necessary. The BSE recommends that the training schedule should be divided into three levels (table).

\section{Schedule}

The basic level will provide a trainee with knowledge of instrumentation, anatomy, and abnormalities sufficient to understand the techniques and applications of echocardiography and to perform clinical studies provided that some supervision is available.

The proficiency level is the central element of the training programme. It contains core sections and adult and paediatric options. At the end of this period the echocardiographer should be able to perform a competent echocardiographic examination appropriate to all the commonly encountered adult and/or paediatric cardiac conditions and write a report of the findings (see subsequent section on reporting of clinical echocardiographic studies)

At the specialised level the echocardiographer should have enough expertise to train other staff to proficiency level. He or she also should have a knowledge of one or more of the more specialised applications of echocardiography - such as, transoesophageal studies and advanced Doppler or fetal techniques. Details of the specialised level syllabus have not yet been worked out. Echocardiographers who have reached the specialised level should be encouraged to keep up with developments by continuing their education and attending postgraduate meetings. We also recommend that each echocardiographer continues to perform at least 100 echocardiographic studies a year to maintain practical proficiency.

\section{Organisation of training}

Practical training in echocardiographic skills will usually be obtained within the trainee's own department under the guidance of an experienced (at least to proficiency level) echocardiographer. Where local facilities are inadequate, the trainee may have to visit a larger centre. It is hoped to accredit reference sites and to provide information on the number of trainees that they can accommodate, the equipment they use, the types of study they perform (adult, paediatric, intraoperative, etc), and any associated charges.

Training courses that provide the opportunity to scan selected subjects under expert practical guidance are invaluable for those who are starting in echocardiography or for those who wish to refine and/or learn new skills. Though such courses will form part of training they do not provide sufficient breadth of scanning experience for a trainee to achieve competence at proficiency level.

Many hospital departments will also be able to provide the bulk of the theoretical training and hold regular echocardiographic tutorials and reporting/review meetings. Trainees should attend these sessions to increase and broaden their knowledge.

Formal training courses will continue to play a major part in delivering training in echocardiographic theory to larger groups. It may be difficult, however, for a trainee to determine which course is most suitable. The BSE has compiled a directory of training courses that provides information on course content in a standardised format and indicates which sections of the syllabus each course aims to cover. It is envisaged that courses that meet BSE criteria will be able to claim accreditation and recommendation. This would be of particular relevance to courses sponsored by manufacturers who wish to demonstrate an educational commitment beyond that of a marketing or sales exercise. Accreditation for courses claiming to meet the syllabus recommendations of the BSE will be based upon retrospective analysis of attendee feedback given in course questionnaires. However, provisional prospective accreditation (for one year only) may be awarded upon examination of the course programme. During the period of provisional accreditation the course will be assessed to decide upon formal accreditation.

We hope that existing or future training courses will be able to accommodate most of the proposed BSE syllabus. If, however, trainees cannot obtain suitable course training, the BSE may have to consider establishing its own independent training courses. Organisers of seminars and conferences etc may apply for "recommendation by the BSE 
for further education in echocardiography". This recommendation will be based upon an examination of the conference programme and faculty content by the Education and Training Subcommittee of the BSE.

\begin{abstract}
Assessment
Implicit in setting out recommendations for a training programme and defining levels of competence is a mechanism for objective assessment of echocardiographic skills. In all professions training and education are followed by assessment of candidates' abilities before they are allowed to practise their skills independently. Echocardiographers (both doctors and non-medical staff) are often required to perform and/or interpret examinations without adequate training. A nationally recognised measure of proficiency in echocardiography would help to avoid this. Furthermore, experience in echocardiography is regarded as an asset when technical and junior medical staff are considered for promotion. An objective measure of this skill would be useful both to the candidates and to their employers. It may be that a qualification in echocardiography will be rated more highly by technical staff than by medical staff. In practice, however, both groups are involved to the same extent in performing echocardiographic studies and it would be wrong to deny either group the opportunity of having their proficiency independently assessed. We propose to make optional assessments available at both basic and proficiency level to both medical and technical staff. We intend that both practical skills and theoretical knowledge will be assessed.
\end{abstract}

\section{BASIC LEVEL}

At the basic practical level candidates should be able to demonstrate a clinical examination and should also have a basic understanding of the echocardiographic signs of common abnormalities. This assessment will be made at an approved reference site. Basic level theoretical assessment, by a series of multiple choice questions set by the BSE Training and Education Committee, could be undertaken on the same occasion as the practical assessment. The examination should take place simultaneously at several sites around the country.

\section{PROFICIENCY LEVEL}

Assessment for the proficiency level will probably take place twice a year at one site. The practical element will be based on a video tape of 10 case studies submitted by the candidate with a written commentary and an optional audio commentary describing the views used and abnormalities seen. Each case should be accompanied by the clinical details at referral and a report written by the candidate. Each trainee should submit a log-book documenting at least 250 studies; this should record whether assistance was required to perform and interpret the studies and must be authenticated by the section head of the echocardio- graphic department. The log-book will resemble that provided by the British Cardiac Society for documenting training in interventional procedures. All candidates must satisfy the Education and Training Subcommittee that the appropriate standard of practical skill has been attained, and they may be required to demonstrate this.

Assessment of theoretical knowledge for the proficiency level will consist mainly of multiple choice questions with sections on physics and instrumentation and on cardiac abnormalities and their associated echocardiographic findings. Videotapes of clinical cases will be used to assess interpretive skills.

\section{SPECIALISED LEVEL}

Proposals for practical or theoretical examination at the specialised level have not yet been formulated.

\section{Exemption}

Experienced echocardiographers will be exempted from basic and proficiency level examinations. Those requesting exemption should submit a curriculum vitae and a questionnaire detailing their experience in echocardiography. If, in the judgment of the Education and Training Subcommittee, further evidence of their proficiency is required, they may be asked to complete the logbook/video section of proficiency assessment. We expect that many current members of the BSE will be eligible for exemption and that all exemptions will be at basic or proficiency level because no criteria have yet been set for the specialised level.

\section{Costs}

Those claiming exemption will be charged $£ 20$ to defray the administrative costs. Those who obtain accreditation by examination will also pay this administration charge plus an additional fee (not yet set) to cover the examination expenses.

\section{Reporting of clinical echocardiographic studies}

Though the accreditation process is optional we recommend that all staff performing and/or reporting on echocardiographic studies should have achieved the proficiency level in adult and/or paediatric studies. We recognise, however, that some may prefer to ensure for themselves that they have adequate practical skills and knowledge of the proficiency level syllabus. Nevertheless the BSE advises members to avail themselves of the more objective measures of proficiency level skills.

The BSE Council further recommends that echocardiographic reports written by nonmedical staff should not proffer a medical opinion on patient management-for example, the report should not directly recommend anticoagulation or surgical intervention. It has been suggested that reports generated by nonmedical staff should be exclusively descriptive 
without any conclusions on pathology, diagnosis, or severity. It is the general view of the BSE Council that this would considerably reduce the value of echocardiography reports to those who are not familiar with echocardiographic interpretation. The report should also specify the position and title of the reporter. The BSE syllabus covers issues such as generating appropriate reports and understanding the clinical context within which such studies may be requested.

Non-medical staff working in a NHS hospital and within the terms of their job description should be indemnified by their employer - that is, the hospital. In addition the BSE recommends that non-medical staff who perform or report unsupervised studies should write to their head of department and to the chief executive of the hospital, making it clear that they are performing and/or reporting unsupervised echocardiographic studies and that unless they are directed to the contrary (in writing) they will assume that the head of department and chief executive agree with this and that they are indemnified by the hospital. Copies of all such correspondence should be kept.

The BSE recommends that staff who perform and/or report echocardiograms out of their departments, especially in private practice, should obtain their own appropriate medical indemnity insurance. The society does not recommend any particular policies, but we know of members who have obtained insurance (through brokers specialising in such policies) from the Medical Defence Union and through the United Kingdom Association of Sonographers.

Some members may feel more comfortable having their own insurance, irrespective of whether or not they are in private practice. This means that they need not rely on their employer to defend any legal action. For example, the hospital may consider it in its own interest to admit liability on behalf of an employee whereas the individual may prefer to defend the case independently.

\section{Conclusion}

The assessments outlined above are entirely optional and will be provided by the BSE. Many echocardiographers wish to measure their knowledge and skills against an objective national standard. We believe that providing them with an opportunity to do so will enhance the value of a recommended training syllabus and will also reflect one of the primary aims of the British Society of Echocardiography - to raise the standards of echocardiography in the United Kingdom and Republic of Ireland.

The BSE training syllabus, the log-book layout, and the forms for application for exemption from accreditation procedures and application for accreditation as a reference site are available from:

Honorary Secretary,

British Society of Echocardiography,

9 Fitzroy Square,

London W1P 5AH (fax 071388 0903)

or telephone Caroline Westgate on 0713528121 